\title{
Clinicopathological study of 50 cases of Dengue
}

\author{
Mane $\mathbf{V}^{1}$, Mohite $\mathbf{S}^{2}$ \\ ${ }^{1}$ Dr. Vaibhav Mane, MD, Associate Professor of Pathology, ${ }^{2}$ Dr. Sushant Mohite M.B.B.S., Junior Resident Dept. of \\ Pathology, Bharati Vidyapeeth Deemed University, Medical College and Hospital, Sangli, Maharashtra, India
}

Address for correspondence: Dr. Vaibhav Mane, Email: vaishnavilab@rediffmail.com

\begin{abstract}
Aim and objective: The objective of this study was to correlate laboratory tests during the evolution of dengue fever, comparing frequencies between the different clinical forms in order to use test results to predict the severity of the disease. Methods: This is an observational, descriptive and retrospective study of 50 patients with clinical and serological diagnoses of dengue fever. The tests analyzed were blood count, platelet count, and serum aspartate aminotransferase (AST) and alanine aminotransferase (ALT) concentrations. Results: Thrombocytopenia and elevated transaminases were observed in patients with classic dengue fever. The main laboratory abnormalities found in dengue hemorrhagic fever were thrombocytopenia, hemoconcentration and elevated transaminases, similar to severe dengue with the exception of hemoconcentration. Most laboratory abnormalities started on the 3rd day but were more evident on the 5 th day with restoration of values by the $11^{\text {th }}$ day; this was more prominent in under 15 -year-olds and with the more severe clinical forms. Conclusion: These results are relevant in assessing the disease because they can be used as markers for more severe forms and can help by enabling the adaptation of the therapeutic conduct to the needs of individual patients.
\end{abstract}

Keywords: Dengue Hemorrhagic Fever, Dengue Shock Syndrome, Leucopenia, Thrombocytopenia

\section{Introduction}

Dengue is caused by one of the four serotypes of the dengue virus (DEN-1, DEN-2, DEN-3 and DEN-4) also referred to as an arbovirus (arthropod-borne viruses) that belongs to the genus Flavivirus of the family Flaviviridae. It is a disease with a wide clinical spectrum and a wide variety of presentations, ranging from asymptomatic to an undifferentiated fever (viral syndrome) to the more severe forms such as severe dengue (SD) or Dengue hemorrhagic fever (DHF).

Transmission to humans occurs by the bite of the female Aedes aegypti mosquito infected by one of four serotypes of the virus.). The period of transmission from humans to mosquitoes begins one day before the start of fever up to the sixth day of illness corresponding to the viremia phase. In humans, the incubation period ranges from 3 to 15 days (intrinsic incubation) with an average of 5 days. The diagnosis of

Manuscript received: $4^{\text {th }}$ Aug 2015

Reviewed: $11^{\text {th }}$ Aug 2015

Author Corrected: $19^{\text {th }}$ Aug 2015

Accepted for Publication: $28^{\text {th }}$ Aug 2015 dengue fever is carried out based on clinical, epidemiological and laboratory data.

Leucopenia is the most prominent hematological change, sometimes with counts of less than $2 \times 10^{3} / \mu \mathrm{L}$. However, there are reports of mild leukocytosis at the onset of the disease, with neutrophilia. Lymphocytosis is a common finding, with the presence of atypical lymphocytes.

Of biochemical variables, the most frequent changes occur in liver function tests such as in serum aspartate aminotransferase (AST), serum alanine aminotransferase (ALT), Gamma-glutamyl transpeptidase and alkaline phosphatase levels, and serum albumin concentrations.

In this context, the present study aimed to assess the biochemical and hematological dynamics of patients with dengue fever in order to increase the sensitivity of the screening by healthcare professionals in the most 
serious cases and to try to identify laboratory markers that may indicate this evolution.

\section{Materials and Methods}

Fifty clinically suspected cases of dengue were studied retrospectively.This case series was conducted in Bharati Medical colloge Sangli And Vaishnavi pathology laboratory Sangli, Maharashtra.

Inclusion criteria: All patients presenting with fever of less than 2 weeks duration, body rashes, flushing and bleeding manifestations were included.

Exclusion criteria: Patients with fever of more than 2 weeks duration, haematological disorders and malignancies, liver failure, peptic ulcer, chronic splenomegaly syndrome, enteric fever and autoimmune disorders, positive for malaria were excluded.

This is an observational, descriptive and retrospective study of 50 patients with clinical and serological diagnoses of dengue fever. The tests analyzed were blood count, platelet count, and serum aspartate aminotransferase (AST) and alanine aminotransferase (ALT) concentrations.

Fifty clinically suspected cases of dengue were studied. Case definition criteria for dengue fever were high fever, fever with rash, retro orbital pain, myalgia, arthralgia, and conjunctival congestion [1]. The criteria for dengue hemorrhagic fever (DHA)[1] included a triad of hemorrhagic manifestations, platelet count of less than $1.0 \mathrm{lakh} / \mathrm{cumm}$ and clinical signs of plasma leakage observed in the form of pleural effusion or ascites.

Hematological examination included complete hemogram with Nihon Kohden 5 part differential cell counter and peripheral blood was evaluated. Hemoconcentration was seen as raised hemoglobin or RBC count. Leucopenia was defined as total WBC count less than 4000/cumm and thrombocytopenia as less than $1.0 \mathrm{lakh} / \mathrm{cumm}$. Liver function tests were done on fully automated biochemistry analyzer.

\section{Results}

Out of 50 cases seropositive for dengue 27(54\%) were positive for NS1, 19 (38 \%)for IgM and 4(8\%) for IgG. Serological test sensitivity was $96-98.9 \%$ \& specificity was $96.2-100 \%$. Out of 50 patients 20 were females and 30 were males.

The clinical, hematological and biochemical features observed are as in Tables 1 to 7

Table 1 : Clinical features in Dengue fever

\begin{tabular}{|l|l|l|l|}
\hline Clinical Features & $\begin{array}{l}\text { NS1 } \\
\mathbf{N = 2 7}\end{array}$ & $\begin{array}{l}\text { IgM } \\
\mathbf{N = 1 9}\end{array}$ & $\begin{array}{l}\text { IgG } \\
\mathbf{N = 4}\end{array}$ \\
\hline High Grade Fever & $22(81 \%)$ & $16(83.2 \%)$ & $02(50 \%)$ \\
\hline Fever With rash & $13(40.3 \%)$ & $10(52 \%)$ & $00(00)$ \\
\hline Myalgia & $19(70.3 \%)$ & $14(72.8 \%)$ & $02(50 \%)$ \\
\hline Arthalgia & $10(37 \%)$ & $04(20.8 \%)$ & $01(25 \%)$ \\
\hline Conjuctival congestion & $11(40.7 \%)$ & $08(41.6 \%)$ & $01(25 \%)$ \\
\hline Hepatomegaly & $05(18.5 \%)$ & $08(41.6 \%)$ & $02(50 \%)$ \\
\hline hepatospleenomegaly & $02(7.4 \%)$ & $02(10.4 \%)$ & $01(25 \%)$ \\
\hline Nausea & $12(44.4 \%)$ & $10(50.2 \%)$ & $02(50 \%)$ \\
\hline Vomiting & $07(25.9 \%)$ & $05(26 \%)$ & $00(00 \%)$ \\
\hline Headache & $15(55.5 \%)$ & $12(62.4 \%)$ & $03(75 \%)$ \\
\hline Swollen lymphnodes & $10(37 \%)$ & $07(36.4 \%)$ & $01(25 \%)$ \\
\hline Retroorbital pain & $15(55.5 \%)$ & $09(46.8 \%)$ & $02(50 \%)$ \\
\hline
\end{tabular}


Table 2 : Haemoglobin in Dengue fever

\begin{tabular}{|l|l|l|l|}
\hline Haemoglobin & $\begin{array}{l}\text { NS1 } \\
\mathbf{N}=\mathbf{2 7}\end{array}$ & $\begin{array}{l}\text { IgM } \\
\mathbf{N}=\mathbf{1 9}\end{array}$ & $\begin{array}{l}\text { IgG } \\
\mathbf{N}=\mathbf{4}\end{array}$ \\
\hline $9.0-11.0$ & $4(14.8 \%)$ & $05(26 \%)$ & $00(00 \%)$ \\
\hline $11.0-13.0$ & $11(40.7 \%)$ & $07(36.4 \%)$ & $02(50 \%)$ \\
\hline $13.0-16.0$ & $12(44.4 \%)$ & $0736.4 \%)$ & $02(50 \%)$ \\
\hline
\end{tabular}

Table 3: Total leucocyte count in Dengue Fever

\begin{tabular}{|l|l|l|l|}
\hline Total Leucocyte count & $\begin{array}{l}\text { NS1 } \\
\text { N= 27 }\end{array}$ & $\begin{array}{l}\text { IgM } \\
\text { N= 19 }\end{array}$ & $\begin{array}{l}\text { IgG } \\
\text { N= 4 }\end{array}$ \\
\hline $7000-10000$ & $02(7.4 \%)$ & $02(10.4 \%)$ & $00(00 \%)$ \\
\hline $4000-7000$ & $07(25.9 \%)$ & $06(31.2 \%)$ & $02(50 \%)$ \\
\hline Less than 4000 & $18(66.6 \%)$ & $11(57.2 \%)$ & $02(50 \%)$ \\
\hline
\end{tabular}

Table 4: Platelet count in Dengue Fever

\begin{tabular}{|l|l|l|l|}
\hline Platelet count & $\begin{array}{l}\text { NS1 } \\
\mathbf{N}=\mathbf{2 7}\end{array}$ & $\begin{array}{l}\text { IgM } \\
\mathbf{N = 1 9}\end{array}$ & $\begin{array}{l}\text { IgG } \\
\mathbf{N}=\mathbf{4}\end{array}$ \\
\hline More than 150000 & $01(3.7 \%)$ & $02(10.4 \%)$ & $01(25 \%)$ \\
\hline $150000-100000$ & $06(22.2 \%)$ & $05(26 \%)$ & $01(25 \%)$ \\
\hline $100000-20000$ & $20(74 \%)$ & $12(62.4 \%)$ & $02(50 \%)$ \\
\hline
\end{tabular}

Table 5: SGOT levels in Dengue fever

\begin{tabular}{|l|l|l|l|}
\hline & NS1 & IgM & IgG \\
$\mathbf{N = \mathbf { 2 7 }}$ & $\mathbf{N = 1 9}$ & $\mathbf{N = 4}$ \\
\hline AST (SGOT) Increased & $22(81.4 \%)$ & $11(57.2 \%)$ & $03(75 \%)$ \\
\hline AST ( SGOT )Normal & $05(18.6 \%)$ & $08(41.6 \%)$ & $01(25 \%)$ \\
\hline
\end{tabular}

Table 6: SGPT Levels in Dengue Fever

\begin{tabular}{|l|l|l|l|}
\hline & NS1 & IgM & IgG \\
$\mathbf{N = 2 7}$ & $\mathbf{N = 1 9}$ & $\mathbf{N}=4$ \\
\hline ALT ( SGPT ) increased & $17(62.9 \%)$ & $12(63.4 \%)$ & $03(75 \%)$ \\
\hline ALT ( SGPT )normal & $10(37.1 \%)$ & $07(36.6 \%)$ & $01(25 \%)$ \\
\hline
\end{tabular}

Table 7: Bilirubin levels in dengue fever

\begin{tabular}{|l|l|l|l|}
\hline & NS1 & IgM \\
$\mathrm{N}=27$ & $\mathrm{~N}=19$ & $\begin{array}{l}\operatorname{IgG} \\
\mathrm{N}=4\end{array}$ \\
\hline Bilirubin increased & $02(7.4 \%)$ & $03(15.6 \%)$ & $00(00 \%)$ \\
\hline Bilirubin normal & $25(92.5 \%)$ & $16(83.2 \%)$ & $04(100 \%)$ \\
\hline
\end{tabular}

Hemoconcentration, Leucopenia, and Thrombocytopenia were chief hematological features of seropositive dengue cases. Peripheral blood smear showed activated lymphocytes.Aspartate aminotransferase and alanine aminotransferase were mildly elevated in significant number of cases. None had any bleeding manifestations

\section{Discussion}

Dengue fever is an infectious disease which is difficult to distinguish from other viruses as there are no specific markers that can diagnose the disease early. Because it is a disease that can evolve with serious consequences and even be fatal, this study aimed at analyzing clinical and epidemiological data and laboratory dynamics in order to try to identify biomarkers that are predictive of severity. 
Dengue is caused by a virus belonging to the flavi viridae family (single stranded, positive, nonsegmented RNA virus). It has four distinct serotypes DEN 1, DEN 2, DEN 3 and DEN 4 $[1,2,4]$. Infection with one serotype confers immunity to only that serotype and hence a person may be infected upto four times $[1,2,4]$. Humans are the main reservoir of dengue virus $[1,2,3]$. Dengue presents as dengue fever, dengue haemorrhagic fever (DHF) or dengue shock syndrome (DSS).[1,2,34,5,].

It has been suggested that baseline microvascular permeability in children is greater than that of adults and this could partly explain, why DHF is more frequent in children $[3,5,, 6,7,8]$. In our study, none of the children had DHF/DSS. This antibody dependant mechanism results in an amplified cascade of cytokines and complement activation causing endothelial dysfunction and consumption of coagulation factors leading to plasma leakage and haemorrhagic manifestations. [2,3,8,9,10 ]The severity of the disease depends on the strain and serotype of the virus, age of the patient and degree of viremia.

The most common clinical feature of dengue in our study was high fever of patients. The fever rash was typically macular or maculopapular, often becoming confluent and sparing small islands of normal skin. The rash was not associated with scaling or pruritus $[1,2,11,12,13$,$] . Pervin et al [13], reported occurrence$ of rash in $33 \%$ of patients. Hepatomegaly was observed in more than $30 \%$ of our patients. Hepatomegaly is more common in patients with secondary infection and some of these may be associated with a increase in liver transaminases. Myalgia was observed in $72 \%$ of patients. Pervin et al, reported myalgia in $84.5 \%$ of patients. Hemoconcentration was seen in more than $50 \%$ of patients [13].

Thrombocytopenia (platelets $<1,00,000 / \mathrm{cmm}$ ) was seen in $80 \%$ of patients. The platelet count in these patients ranged between 4,000-1,00,000/cmm. None had any bleeding manifestations. Platelet count was evaluated by Nihon Kohden 5 part diferential cell counter as well as on peripheral blood smear. The counter gives a false low reading when large platelets are present. Such cases were obviated by assessment of platelets on smear. Ratagiri et al [14], reported thrombocytopenia in $82 \%$, DHF in $60 \%$, DSS in $22 \%$ and DF in $18 \%$ of patients. Our study on the other hand reflected DF in $96 \%$.

Leucopenia was observed in $26 \%$ of patients by Ratagiri et at [14]. Leucopenia was observed in more than $60 \%$ of patients in our study. Development of antibodies potentially cross-reactive to plasminogen (due to a similarity in 20 amino acid sequence of dengue $\mathrm{E}$ glycoprotein and a family of clotting factors) could have a role in causing haemorrhage in DHF $[9,11,13]$. The increased destruction or decreased production of platelets could result in thrombocytopenia. Virus-antibody complexes have been detected on the platelet surface of DHF patients suggesting a role for immune-mediated destruction of platelets [14]. The release of high levels of platelet-activating factor by monocytes with heterologous secondary infection may explain the haemorrhage, given that platelet-activating factor may induce platelet consumption and augment adhesiveness of vascular endothelial cells resulting in thrombocytopenia [12]. The presence of $1 \mathrm{gM}$ antibodies in the sera DHF cases that cross-reacted with platelets has been demonstrated $[1,3,6,9]$. These autoantibodies could be involved in the pathogenesis of the disease. IgM and $\operatorname{IgM}$ antibodies assay by ELISA is the commonest diagnostic test. The test based on an increase in the IgG titre by a factor of four is difficult in routine clinical care because a second blood sample is required at the convalescent stage. Cross reactions with other flaviviruses interfere with serologic testing, particularly the ELISA for IgG and this affects the interpretation of test results in travellers exposed to other flavivirus infections,including those previously vaccinated against flavivirus infections, such as yellow fever and Japanese encephalitis [7,13,14]. Rheumatoid factor may lead to an IgM capture assay that is false positive for dengue and like many other flavivirus infections (albeit lesser than with dengue IgG assays) [8].

Primary infections are characterised by an increase in dengue-specific NS1 antigen and IgM antibodies four to five days after the onset of fever and by an increase in IgG antibodies only after seven to ten days. IgM antibodies are detectable for three to six months, whereas IgG antibodies remain detectable for life. In secondary infections, the level of $\operatorname{IgM}$ antibodies is lower than in primary infections and the antibodies are sometimes absent, whereas levels of $1 \mathrm{gG}$ antibodies rise rapidly in secondary infections, even during the acute phase. Thus, the presence of high titers 
of $1 \mathrm{gG}$ early in the course of the disease is a criterion for secondary infection. The sensitivity of $1 \mathrm{gM}$ ranges from $90-97 \%$ as compared with the gold standard haemagglutination-inhibition test. Some false positive reactions can be observed in less than $2 \%$ of cases and a low or negative $\operatorname{IgM}$ reaction in secondary infections.

This study shows that DSS is an uncommon manifestation of dengue virus infection.Dengue infection is generally self limiting. Patients with bleeding manifestations usually have decreased platelet count and leucocyte count. Hemoconcentration, leucopenia, thrombocytopenia, raised AST, raised ALT and plasmacytoid lymphocytes in peripheral blood smear shall give enough clue to test for Dengue serology so as to reduce the morbidity and mortality due to this disease.

According to World Health Organization (WHO),[1,2,3] PCR is a powerful method to be used for dengue diagnosis, but PCR is not widely available and it still needs to be better standardized

\section{Conclusion}

Dengue fever evolves with laboratory alterations starting on the $3^{\text {rd }}$ day and becoming most evident on the $5^{\text {th }}$ day with values restored to normal by the $11^{\text {th }}$ day. The disease was more severe in individuals aged 15 years and older with a more pronounced and persistent presence of liver abnormalities (AST, ALT) and hemoconcentration. The study results are relevant in the characterization of biological markers in the evolution of the disease and can be used as markers for the most severe forms thereby enabling early help with the adaption of therapeutic conduct for specific patients

The results of this study have highlighted the importance of history, clinical examination and the triad of thrombocytopenia, raised hematocrit and elevated liver enzymes for the early diagnosis of DHF without waiting for dengue serology for diagnosis which takes 2-3 days. Therefore, it is recommended that the diagnosis of dengue fever should be made early on the basis of basic investigations. Timely diagnosis and early fluid and supportive therapy reduces the morbidity and mortality from DHF.

Funding: Nil, Conflict of interest: Nil, Permission from IRB: Yes

\section{References}

1. World Health Organization Dengue: Guidelines for diagnosis, treatment, prevention and control. Geneva: WHO; 2009

2. Monath TP. Dengue: the risk to developed and developing countries. Proc Natl Acad Sci U S A. 1994 Mar 29;91(7):2395-400.

3. Lee VJ, Lye DC, Sun Y, Fernandez G, Ong A, Leo YS. Predictive value of simple clinical and laboratory variables for dengue hemorrhagic fever in adults.

J Clin Virol. 2008 May;42(1):34-9. doi: 10.1016/j.jcv.2007.12.017. Epub 2008 Feb 20.

4. Gubler DJ. Dengue and dengue hemorrhagic fever. Clin Microbiol Rev. 1998 Jul;11(3):480-96.

5. Gubler DJ, Meltzer M. Impact of dengue/dengue hemorrhagic fever on the developing world. Adv Virus Res. 1999;53:35-70.

6. Ageep AK, Malik AA, Elkarsani MS. Clinical presentations and laboratory findings in suspected cases of dengue virus. Saudi Med J. 2006 Nov;27(11):1711-3.

7. Guzmán MG, Kourí G. Dengue: an update. Lancet Infect Dis. 2002 Jan;2(1):33-42.

8. Gibbons RV, Vaughn DW. Dengue: an escalating problem. BMJ. 2002 Jun 29;324(7353):1563-6.

9. Gubler DJ. The global pandemic of dengue/dengue haemorrhagic fever: current status and prospects for the future. Ann Acad Med Singapore. 1998 Mar;27(2):227-34.

10. Hober D, Nguyen TL, Shen L, et al. Tumor necrosis factor alpha levels in plasma and wholeblood culture in dengue infected patients: relationship between virus detection and pre- existing specific antibodies. J Med Virol 1998; 54:210-8.

11. Guzmán MG, Alvarez M, Rodríguez R, Rosario D, Vázquez S, Vald s L, Cabrera MV, Kourí G. Fatal dengue hemorrhagic fever in Cuba, 1997. Int J Infect Dis. 1999 Spring;3(3):130-5.

12. Waterman SH, Gubler DJ. Dengue fever. Clin 
Dermatol. 1989 Jan-Mar;7(1):117-22.

13. Pervin M., Tabassum S, Ali M, Kazi M. Dengue Bulletin 2004; 28.
14. Ratageri VH, Shepur TA, Wari PK, Chavan SC, Mujahid IB, Yergolkar PN. Clinical profile and outcome of Dengue fever cases. Indian J Pediatr. 2005 Aug;72(8):705-6.

\section{How to cite this article?}

Mane V, Mohite S. Clinicopathological study of 50 cases of Dengue. Int J Med Res Rev 2015;3(8):794-799. doi: 10.17511/ijmrr.2015.i8.149. 